\title{
SDU
}

\section{Simple Social Choice Rules for Exchange}

by

\section{Ryan Tierney}

Discussion Papers on Business and Economics

No. $4 / 2021$

FURTHER INFORMATION

Department of Business and Economics

Faculty of Business and Social Sciences University of Southern Denmark Campusvej 55, DK-5230 Odense M 


\title{
SIMPLE SOCIAL CHOICE RULES FOR EXCHANGE
}

\author{
RYAN TIERNEY
}

\begin{abstract}
We study the classical problem of trade in two-dimensional Euclidean space. It is known that there is no efficient rule for this model that is compatible with dominant strategy incentives, that is, there is no efficient and strategy-proof rule. We observe that, in addition to incentive constraints, informational constraints are also unavoidable for social planners. Thus, we impose the requirement that finite dimensional messages be sufficient information to realize a rule. In addition, we impose the minimal fairness axioms of anonymity and a weakening of non-bossiness, as well as continuity. The result is a class of rules that is similar to those characterized by Barberà and Jackson ["Strategy-proof exchange", Econometrica, 63 (1995), 51-87].

Key words: Strategy-proof exchange, communication complexity.

JEL codes: D44, D47, D51, D83
\end{abstract}

\section{INTRODUCTION}

Revelation mechanisms are impossible to execute on large preference spaces. This is certainly true of the classical domain, the space of monotone and convex preferences over Euclidean space. Even if agents have preferences in this domain, they cannot communicate them in finite time. We are thus lead to study the category of encoded revelation mechanisms, introduced by Mount and Reiter (1996). These are mechanisms that ask agents to report not their preference relation but rather a simpler message. Presumably, we are running a mechanism in the hope of realizing a social choice rule with desirable properties. Thus, the message must contain all the information that the social choice rule requires. Therefore, if we want to work with simple messages, we must limit the amount of information our rule requires.

Date: Wednesday $10^{\text {th }}$ March, 2021. 
Mount and Reiter (1996) showed that for each social choice rule there is a space of messages that is minimal in the sense that any smaller space would fail to encode all the information the rule requires. This is the essential message space. We study the rules whose essential message space can be embedded in finite dimensional Euclidean space.

We are interested in social choice rules for the exchange of divisible commodities. Each agent is entitled to their endowment and the social choice rule may or may not recommend a trade that increases welfare. For agents to be willing to disclose information about their preferences, it is necessary for the social choice rule to be incentive compatible in some way. We choose dominant strategy incentive compatibility, also known as strategy-proofness. It is well known that in economies of finitely many agents, we cannot expect such rules to be Pareto efficient while still respecting private property (Serizawa and Weymark (2003); Zhou (1991)), so we forego efficiency as a desideratum. We also impose anonymity for two principal reasons: 1) in practice, agents will be wary of participating in a discriminatory mechanism if there are other mechanisms to choose from and 2) because it should be easier to understand the informational demands of a rule when these are the same for all participants. For technical tractability, we also impose a weakening of non-bossiness. Non-bossy non-traders requires that if an agent is not permitted to trade, and if a change in this agent's message still has them not trading, then this change of message has no effect on the other agents. This eliminates from contention rules that set agents aside and have them take the role of a moderator of some kind. Note that anonymity does not necessarily eliminate such rules because the choice of moderator may be based on preferences alone. Given that the preference space is infinite dimensional, there is ample freedom for such rules to operate.

Finally, we limit our study to social choice rules that are continuous (with respect to the topology of closed convergence (Hildenbrand, 1974)). This seems necessary to properly measure information. Note that the informational requirements of a rule are manifested in the partition induced by pre-images of singletons: each of these is the set of economies in which the rule makes the same choice, and so a single message suffices for all such 
economies. Absent any regularity condition, rules may induce arbitrary partitions on the space of economies and so may result in mechanisms that technically can be communicated in finite dimensions but whose message functions are infinitely convoluted. This trades one form of complexity for another.

We also find justification for continuity in normative principles. Note first that, for a strategy-proof rule, each agent at each economy gets their favorite alternative from a set of alternatives that is determined by the other agents. By Berge's theorem of the maximum, therefore, an agent's own allocation should be mostly continuous in their own message, with exceptions arising from non-convex option sets. Since we have a convex consumption space, it seems reasonable the we should have convex option sets, and so beyond this requirement, continuity really imposes that one's influence upon another be continuous.

You and Juarez (2021) provide background literature on the question of simplicity for the design of allocation mechanisms for divisible goods. Theirs is also (to the author's knowledge) the only recent work to study the question. They work in the standard mechanism design environmentquasilinear preferences, unconstrained transfers - and focus on achieving efficiency and incentive compatibility via one dimensional reports. Among other things, they discover that partial Nash implementation is only possible via Vickrey-Clarke-Groves-like (VCG-like) mechanisms and that full implementation is impossible on the full domain; however, they identify a subdomain on which it is. In this manuscript, I allow for arbitrary finitedimensional messages, but I must forego efficiency as I study a pure-exchange environment; there may be no flows of resources into or out of the economy. It is then not surprising that the mechanisms of Barberà and Jackson (1995) should emerge in my study. Strictly speaking, their mechanisms are a special case of the class I find here, but they nonetheless contain the most salient feature of the general class, namely, that trading be ex-ante constrained to a finite number of line segments in the consumption space. 


\section{MODEL}

2.1. Consumption and Preference Spaces. We study social choice allocation rules on a standard preference domain augmented with Leontief preferences. The set of agents is $N$. There is a social endowment $|N| \cdot \omega \in \mathbb{R}^{2}$ and we assume that each agent is endowed with an equal share, $\omega$. The analysis below does not depend on the equality of endowments; it is for ease of exposition. The set of alternatives, $Z$, is the hyperplane given by $\sum_{i \in N} x_{i}=|N| \cdot \omega$, restricted to the non-negative orthant $\mathbb{R}_{+}^{2 \times N}$. Each projection $Z_{i} \subseteq \mathbb{R}_{+}^{2 \times\{i\}}$ is agent $i$ 's personal consumption. Preferences are such that consumption has no externalities. Let $\mathcal{R}_{i}$ be the set of agent $i$ 's hypothetical preferences over his consumption space $Z_{i}$, so the graph of each $R_{i} \in \mathcal{R}_{i}$ is a subset of $Z_{i} \times Z_{i}$. Since $Z_{i}$ and $Z_{j}$ are equivalent, we may assume that for each pair $i, j \in N, \mathcal{R}_{i}=\mathcal{R}_{j}:=\mathcal{R}$. In particular, $\mathcal{R}$ is the set of (weakly) convex and (weakly) monotone preference relations $1^{1}$ A social choice rule, or rule for short, is a function $\varphi: \mathcal{R}^{N} \rightarrow Z$.

A Leontief preference relation is given by a certain kind of monotone, terminal path from the origin. An path is a continuous function $c:[0,1] \rightarrow$ $\mathcal{X}$, where $\mathcal{X}$ is a topological space, for now $\mathbb{R}^{2}$. A path is monotone if $t^{\prime} \geq t$ implies $\left.c\left(t^{\prime}\right) \geq c(t)\right]^{2}$ A monotone path is increasing if $t^{\prime}>t$ implies $c\left(t^{\prime}\right)>$ $c(t)$. A monotone path is terminal if there is $k \in\{1,2\}$ with $c_{k}(1) \geq|N| \omega_{k}$, making the path hit the upper $\geq$-envelope of the consumption space. Each Leontief preference relation $L^{c}$ is generated by a monotone terminal path $c$; for each $\lambda \in[0,1]$, the set $\{y: y \geq c(\lambda)\}$ is the upper contour set of $L^{c}$ at $c(\lambda)$. Not every monotone terminal path generates a Leontief relation; consider a piecewise linear path that initially has the form $\lambda \mapsto \lambda(1,1)$ and then has the form $\lambda \mapsto \lambda(0,1)$. More generally, let $\mathcal{C}$ be the set of terminal paths $c$ such that, $c(0)=0$ and if $c\left(t^{\prime}\right)>c(t)$, then for each $t^{\prime \prime}>t^{\prime}$,

\footnotetext{
${ }^{1}$ As usual, $R_{i}$ is strictly monotone if $x \gtrless y$ implies $x R_{i} y$ and not $y R_{i} x$. It is strictly convex if its upper contour sets are strictly convex.

${ }^{2}$ Notational conventions: Let $\geq$ and $>$ be the standard relations on $\mathbb{R}$. For $\{x, y\} \subset \mathbb{R}^{2}$, $x \geq y$ if and only if $x_{1} \geq y_{1}$ and $x_{2} \geq y_{2}$. Similarly, $x>y$ if and only if $x_{1}>y_{1}$ and $x_{2}>y_{2}$.
} 
$c\left(t^{\prime \prime}\right)>c\left(t^{\prime}\right)$. Then it is easy to verify that $\mathcal{C}$ is the set of all paths that generate Leontief preferences.

For $q \in \mathbb{R}_{+}$, denote by $h(q)$ the line in $\mathbb{R}^{2}$ with normal $(q, 1)$ and passing through $\omega$. For $q \in \mathbb{R}_{+}$, each preference relation $R_{i} \in \mathcal{R}$, when restricted to $h(q)$, has a unique ideal point $p\left(R_{i} ; q\right)$, which we shall call its peak on $h(q)$. Restricting attention to allocations on $h(q)$, the problem becomes a variant of the well-studied single-peaked allocation problem (see Sprumont (1991)). A rationing rule on $h(q)$ is a function $\varphi^{q}: \mathcal{R}^{N} \rightarrow(h(q))^{N}$ such that $\varphi^{q}$ is a rule.

2.1.1. The topology of the space of preferences. Given $R_{i} \in \mathcal{R}$ and $x \in Z_{i}$, there is $y \in Z_{i}$, on the diagonal, such that $x R_{i} y R_{i} x$, which is to say that $x$ and $y$ are $R$-indifferent. Let $u_{i}(x)=\|y\|$. The utility function $u_{i}$ thus constructed represents $R_{i}$. Given this representation mapping, we may define the following distance on the subset $\mathcal{R}^{\circ}$ of strictly monotone preferences in $\mathcal{R}$ :

$$
d\left(R_{i}, R_{i}^{\prime}\right)=\max _{x \in Z_{i}}\left\|u_{i}(x)-u_{i}^{\prime}(x)\right\|
$$

Kannai (1970) showed that this metric induces the coarsest topology that makes sets of the form $\{(x, y, R): x R y\}$ open in $\left.Z_{i} \times Z_{i} \times \mathcal{R}^{\circ}\right\}^{3}$ Inspection of Kannai's proof reveals that it works equally well on $\mathcal{R}$, making $(\mathcal{R}, d)$ a metric space. Hildenbrand (1970) then demonstrated that $(\mathcal{R}, \tau)$, with $\tau$ the topology induced by $d$, is a subspace of the space of all reflexive binary relations with the topology of closed convergence. This latter space is compact, making closed subsets of $\mathcal{R}$ compact, and their images under a continuous function likewise compact. In sum: a continuous function on $(\mathcal{R}, d)$ is a closed map. This observation is the key property we require of the topology.

${ }^{3}$ Kannai actually worked with metric

$$
d^{\prime}\left(R_{i}, R_{i}^{\prime}\right)=\max _{x \in Z_{i}} \frac{\left\|u_{i}(x)-u_{i}^{\prime}(x)\right\|}{1+\|x\|^{2}} .
$$

This is easily seen to be equivalent to $d$ as $d^{\prime} \leq d \leq\left(1+|N|^{2}\|\omega\|^{2}\right) d^{\prime}$. 
We henceforth assume $\mathcal{R}$ is endowed with topology $\tau$ and associated metric $d$. We likewise identify $\mathcal{C}$ and the set of Leontief preferences, so topologize $\mathcal{C}$ as a subspace of $\mathcal{R}$. First note that our utility representation gives, for Leontief preference $L^{c}$,

$$
u(x)=\sqrt{2} \max _{c(\lambda) \leq x} \max \left\{c_{1}(\lambda), c_{2}(\lambda)\right\}
$$

This then induces a metric between paths in $\mathcal{C}$ that finds the maximum either horizontal or vertical distance between them. We present that metric formally in Section A, where we also prove the following proposition.

Proposition 1. The topology that $(\mathcal{R}, \tau)$ induces on $\mathcal{C}$ is metrizable and has infinite Lebesgue covering dimension.

The significance of the latter claim lies in the fact that $\mathcal{C}$ does not immediately admit a proof that it cannot be embedded in $\mathbb{R}^{k}$. Firstly, $\mathcal{C}$ being equipollent with the set of monotone continuous functions, it actually has the cardinality of the continuum. Second, while there is an obvious convex structure that can be put on $\mathcal{C}$, and so we may hope show it has infinite dimension as a topological vector space, the vector operations are not in fact continuous with respect to our topology, as the example below shows.

Note that if two paths converge in both their maximal horizontal and vertical distance, they converge in Hausdorff distance. The converse is not true, as can be seen by considering a sequence of paths of the form $\lambda \mapsto$ $\lambda\left(1, \frac{1}{n}\right)$. Thus, the topology on $\mathcal{C}$ is strictly finer than that induced by the Hausdorff distance.

2.2. Encoded Revelation Mechanisms. Mount and Reiter (1996) introduce encoded revelation mechanisms to study the complexity of social choice rules. Their idea is to capture via an intermediate message space the way a social choice rule uses information. We instead put a constraint on the message space and deduce the class of rules that meet this restriction. Whereas Mount and Reiter studied techniques for deriving a lower bound on the number of variables that a rule uses, we instead require that a rule uses at most finitely many variables. 
Definition. Fix a rule $\varphi$. Let $\mathcal{M} \stackrel{\text { def }}{=} \times_{i \in N} \mathcal{M}_{i}$ be a set and $\sigma$ a list of continuous functions $\sigma_{i}: \mathcal{R}_{i} \rightarrow \mathcal{M}_{i}$. If there exists a function $\varphi^{*}$ such that the graph

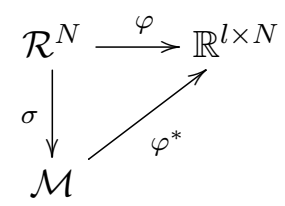

commutes, then the triple $\left(\mathcal{M}, \sigma, \varphi^{*}\right)$ is an encoded revelation mechanism that realizes $\varphi$. We call $\sigma$ the message function of the mechanism and say that $\sigma$ encodes $\mathcal{R}^{N}$. We also call $\mathcal{M}$ an encoded revelation message space.

Let $\varphi$ be a rule and consider a pair of preference relations $R_{i}, R_{i}^{\prime} \in \mathcal{R}_{i}$. If for each profile $R$ this pair satisfies,

$$
\varphi\left(R_{i}, R_{-i}\right)=\varphi\left(R_{i}^{\prime}, R_{-i}\right)
$$

then agent $i$ need not distinguish $R_{i}$ and $R_{i}^{\prime}$ when playing the revelation game induced by the rule; they are informationally equivalent. The quotient of $\mathcal{R}_{i}$ under this equivalence $\stackrel{\varphi}{\sim}_{i}$ is the space of $i$ 's characteristics that the rule actually uses, all other information is discarded. The encoded revelation mechanism that uses these quotients as message spaces is called the essential revelation mechanism. Intuitively it is the encoded revelation mechanism that uses the smallest message space.

Implicit in the definition of encoded revelation mechanisms is the preservation of each agent's privacy. The alternative construction would be to have agents first pool their information, but this would pass the computational burden of the rule entirely to the agents. Consider the smallest message space arising from this approach. It would use the equivalence relation defined as follows: profiles $R$ and $R^{\prime}$ are equivalent, denoted $R \approx R^{\prime}$, if $\varphi(R)=\varphi\left(R^{\prime}\right)$. In this case, $\mathcal{R}^{N} / \approx$ would be bijective with the image of $\varphi$. Moreover, if $\varphi$ is continuous, $\mathcal{R}^{N} / \approx$ and $\varphi\left(\mathcal{R}^{N}\right)$ would be topologically equivalent. Thus, the problem would be trivialized 4

${ }^{4}$ Endow $\mathcal{R}^{N} / \approx$ with the quotient topology. Let $P$ be the projection map from $\mathcal{R}^{N}$ to the quotient space. The map $\varphi^{*}$ given by $\varphi^{*} \circ P=\varphi$ is continuous since $\varphi$ is continuous (See 
In the class of privacy preserving mechanisms, encoded revelation mechanisms are more general than revelation mechanisms but are obviously not the most general. It would have been preferable, for example, to consider communication protocols that allow for multistage interaction, as in Nisan and Segal (2006). This approach has proven prohibitive at present, possibly because our goal differs. Whereas we impose an upper bound on information, Nisan and Segal (2006) study the minimal information required to calculate efficient allocations.

2.3. Properties of Rules. Properties of primary interest in the literature are strategy-proofness and anonymity. As these are standard, we will not dwell on them.

Strategy-proofness: $\forall R \in \mathcal{R}^{N}, \forall i \in N, \forall R^{\prime} \in \mathcal{R}, \varphi_{i}(R) R_{i} \varphi_{i}\left(R_{i}^{\prime}, R_{-i}\right)$.

Anonymity: $\forall$ bijections $\pi: N \rightarrow N, \forall R \in \mathcal{R}^{N},\left(\varphi_{\pi(i)}(R)\right)_{i \in N}=\varphi\left(\left(R_{\pi(i)}\right)_{i \in N}\right)$.

Since we study the exchange of privately owned commodities, we must make formal the notion of private ownership. This is particularly simple given the assumption that all agents have identical endowment $\omega$.

Voluntary Participation: $\forall R \in \mathcal{R}^{N}, \forall i \in N, \varphi_{i}(R) R_{i} \omega$.

Rules that satisfy voluntary participation are called exchange rules.

The next property formalizes our limitation on the complexity of the rule. We require that the rule depend on at most finitely many real variables, making a finite dimensional vector sufficient to communicate an agent's interests. While restrictive, this is still far more information than is feasible in reality, as it allows for arbitrary precision.

Simplicity: There exists an encoded revelation mechanism $\left(\mathcal{M}, \sigma, \varphi^{*}\right)$ such that $\mathcal{M}$ may be embedded in finite dimensional Euclidean space.

We require a rule to be continuous in the topology of closed convergence. This is a metrizable topology important in the foundations of general equilibrium (Hildenbrand, 1974). More importantly, the convergence of preference

Kelley (2008) Theorem 3.9). As mentioned, $\varphi^{*}$ is bijective. We show in the appendix that $\varphi$ is a closed map. Thus, for closed $U \subseteq \mathcal{R}^{N} / \approx, \varphi^{*}(U)=\varphi\left(P^{-1}(U)\right)$ is closed, making $\varphi^{*}$ a homeomorphism. 
relations in this topology is what we expect it should be: upper contour sets get closer and closer.

Continuity: Endow $\mathcal{R}$ with the topology of closed convergence and assume that $\mathbb{R}$ and any of its products are always endowed with the usual topology. Then $\varphi$ is continuous from $\mathcal{R}^{N}$ to $\mathbb{R}^{l \times N}$.

Anonymity prevents a rule from discriminating based on the names of agents. However, it still allows the rule to discriminate based on any element of the agent's preference relation, however questionable the relevance of that part. We may imagine that, in practice, some idiosyncrasies of an agent's behavior, which proceed from idiosyncrasies in his preferences, may identify him even when his identity is obscured. Note that $\mathcal{R}$ is infinite dimensional, so there is ample space for this. The rule might then become arbitrary. We therefore put a very weak limitation on this, preventing this sort of responsiveness only when the agent is not participating.

Non-bossy Non-traders: Suppose that $\varphi_{i}\left(R_{i}, R_{-i}\right)=\varphi_{i}\left(R_{i}^{\prime}, R_{-i}\right)=\omega$. Then for each $j \in N, \varphi_{j}\left(R_{i}, R_{-i}\right)=\varphi_{j}\left(R_{i}^{\prime}, R_{-i}\right)$.

\section{Results}

Recall that a rationing rule chooses, a priori, a line through $\omega$ in which to restrict its domain. In other words, a rationing rule takes the original problem and simplifies it so that it can be viewed as a single-peaked division problem as in Sprumont (1991). We show that a rule satisfying our properties must be composed of at most $|N|-1$ rationing rules. The particular profile of preferences will determine which rationing rule is deployed.

Formally, we show that the above conditions imply a subset of the following class:

Barberà \& Jackson (B\&J) Rules: Each rule $\varphi$ is given by a non-decreasing list of $|N|-1$ real numbers and $|N|-1$ strategy proof rationing rules. Let $Q:=\left(q^{1}, q^{2}, \ldots, q^{|N|-1}\right)$ be one such list of numbers. Let $\Phi:=\left(\varphi^{1}, \ldots, \varphi^{|N|-1}\right)$ be a list of rationing rules, where each $\varphi^{k}$ is a rationing rule on $h\left(q^{k}\right)$ and if $q^{k}=q^{k-1}$ then $\varphi^{k}=\varphi^{k-1}$. Let $q^{k^{*}} \in Q$ satisfy $k^{*}=\left|\left\{i \in N: p_{1}\left(R_{i} ; q^{k^{*}}\right) \geq \omega_{1}\right\}\right|$. Then $\varphi(R)=\varphi^{k^{*}}(R)$. 
Theorem 1. Assume the exchange rule $\varphi$ satisfies strategy-proofness, anonymity, continuity, simplicity, and non-bossy non-traders. Then $\varphi$ is a continuous Barbera \& Jackson rule.

In their paper, Barberà and Jackson (1995) (henceforth B\&J) characterize a smaller class of rules. They show that a strategy-proof, anonymous, voluntary, non-bossy, and tie-free rule is a Barberà \& Jackson Rule that uses uniform rationing. This means that all agents on a given side of the market who are constrained by the rationing are consuming the same quantity. Our rules are both more and less restricted. We require continuity, where B\&J do not. This means that if a rule transitions from $q^{k}$ to $q^{k+1}$, and if $q^{k} \neq q^{k+1}$, then all agents must first converge to $\omega$. We give an example of such a rule below. However, by weakening non-bossiness, we obtain a great deal more freedom. In particular, B\&J show that, in this model, strategy-proofness and non-bossiness together imply the weak form of group-strategy-proofness.

It should be noted as well that tie-freeness is in fact a weak continuity axiom. It implies, given convex preferences, that an agent is indifferent between two messages if and only if they yield him the same consumption bundle. This further implies that each agent's own consumption varies continuously with his own message. Thus, our continuity here only adds that one's effect on other agents be continuous.

3.1. Examples, and some new rules for single-peaked rationing. As previously noted, when the consumption space is restricted to a line $h(q)$, we arrive at a model that is a variant of Sprumont's (1991) rationing model. We have discovered that rules satisfying our properties must indeed be constructed from several such rationing rules. Thus, to give concrete examples of B\&J rules, we focus, in this section, on the rationing model.

Each agent $i$ is to consume a quantity $\xi_{i} \in \mathbb{R}$, and a feasible allocation $\xi \in \mathbb{R}^{N}$ is such that $\sum_{i} \xi_{i}=0$. There are upper and lower bounds $\underline{b}<0<\bar{b}$ on the consumption space. Note that if the bounds are set appropriately, this consumption space may be isometrically mapped to $h(q) \cap \mathbb{R}_{+}^{2}$ such that 0 maps to $\omega$. Now if $q>0$ then each $R_{i}$ restricted to $h(q)$ is a convex 
preference relation having a unique favorite point $p\left(R_{i}\right)$, called its peak (note we suppress reference to $q$ in $p$ here, as the former will remain constant in this section).

The rules we construct will be calculated first by applying a transformation on the profile $\left(p\left(R_{i}\right)\right)_{i \in N}$ of peaks and then operating the Uniform Rule Sprumont, 1991) on the derived profile. A profile $\hat{p} \in \mathbb{R}^{N}$ of peaks - derived or primitive - naturally divides into two sides of the market, $N^{-}(\hat{p})=\left\{i \in N: \hat{p}_{i}<0\right\}$ and $N^{+}(\hat{p})=\left\{i \in N: \hat{p}_{i}>0\right\}$. Given a side of the market $\circ \in\{-,+\}$,

$$
\Omega^{\circ}(\hat{p})=\sum_{i \in N^{\circ}(p)}\left|\hat{p}_{i}\right|
$$

is the offer implied by these peaks that this side makes to the other. The short side of the market is the one making the smaller offer. Under the Uniform Rule, the members of the short side of the market get their peaks. There is then a unique $\lambda \in \mathbb{R}$ for the long side such that it is feasible to give all long side agents $i$ with $\left|\hat{p}_{i}\right|<|\lambda|$ exactly $\hat{p}_{i}$ and the remaining agents $\lambda$.

To arrive at our most basic rule, which we shall, with abuse of terminology, simply call the Uniform rule, the derived profile of peaks is found by the mapping

$$
R \mapsto\left(\operatorname{med}\left\{\underline{b}, p\left(R_{i}\right), \bar{b}\right\}\right)_{i \in N} .
$$

That is, this simply ensures that the result of the rule can be mapped properly to the original two-dimensional space.

More generally, we shall have a continuous function that maps each peak to a number that is larger in magnitude, and then each agent's derived peak will be constrained by these functions applied to the other agents. Formally, assume $f: \mathcal{R} \rightarrow \mathbb{R}$ is a continuous function such that $\left|f\left(R_{i}\right)\right| \geq\left|p\left(R_{i}\right)\right|$, $f\left(R_{i}\right) p\left(R_{i}\right) \geq 0$, and such that $p\left(R_{i}\right)=0$ implies $f\left(R_{i}\right)=0$. Then agent $i$ 's derived peak is $p\left(R_{i}\right)$ if $i$ is on the short side and

$$
\hat{p}_{i}(R)=\operatorname{sign}\left(p\left(R_{i}\right)\right) \min \left(\left\{\left|f\left(R_{j}\right)\right|: p\left(R_{j}\right) p\left(R_{i}\right) \geq 0\right\} \cup\left\{\left|p\left(R_{i}\right)\right|\right\}\right)
$$


if $i$ is on the long side. We call rules thus calculated Collapsing Uniform Rules.

Proposition 2. Collapsing Uniform Rules are strategy-proof, anonymous, continuous and respect voluntary participation.

Proof. Anonymity is clear. Voluntary participation follows from the fact that an agent is given an amount that is (weakly) between their peak and 0 , and since preferences are convex, this is at least as good as 0 .

We shall first show that the Uniform Rule is continuous on $\mathbb{R}^{N} 5$ Considering first $\hat{p} \in \mathbb{R}^{N}$ such that no agent has $\hat{p}_{i}=\lambda$, we can clearly see that, locally, $\lambda$ is calculated as

$$
\frac{\sum_{\xi_{j}=\hat{p}_{j}} \hat{p}_{j}}{\left|\left\{k: \xi_{k} \neq \hat{p}_{k}\right\}\right|},
$$

and so is locally continuous. This further allows us to conclude that peak profiles of this sort form an open set. They also form a dense subset of $\mathbb{R}^{N}$ : for each $i$ with $\hat{p}_{i}=\lambda$, perturb $\hat{p}_{i}$ by $\varepsilon$ so that $\left|\hat{p}_{i}+\varepsilon\right|>\left|\hat{p}_{i}\right|$. Thus, $i$ starts as being rationed and remains rationed and clearly $\lambda$ does not change in response. In sum, there is an open and dense set of peak profiles $\hat{p} \in \mathbb{R}^{N}$ such that $\lambda$ is calculated via line 3.1, and so the Uniform Rule is continuous on this set, which we denote $\mathcal{P}$.

Given $\hat{p} \in \mathbb{R}^{N}$, there is a sequence $\hat{p}^{n} \in \mathcal{P}$ converging to it. Let $\lambda^{n}$ be the sequence of thresholds induced by $\hat{p}^{n}$. By choosing a subsequence, if necessary, we may assume $\hat{p}_{i}^{n} \hat{p}_{i}^{n+1}$ and $\lambda^{n} \lambda^{n+1}$ remain non-negative. Assume further that $\lambda^{n} \geq 0$; the opposite case is symmetric. By choosing a further subsequence, we may also assume that, for each $i$, either $\hat{p}_{i}^{n}<\lambda^{n}$ or $\hat{p}_{i}^{n}>\lambda^{n}$ on the entire sequence. Thus, by inspecting line 3.1, we see that $\lambda^{n}$ is convergent, with limit $\lambda$. Since the feasibility constraints yield a closed set, $\lambda$ induces a feasible allocation and, moreover, $\hat{p}_{i}^{n}<\lambda^{n}$ implies $\hat{p}_{i} \leq \lambda$ and $\hat{p}_{i}^{n}>\lambda^{n}$ implies $\hat{p}_{i} \geq \lambda$. It follows then by the construction of the

Sprumont (1991), on the path to characterizing the Uniform rule, showed that it is separately continuous. 
Uniform Rule, since only one threshold satisfies feasibility and the required inequalities, that $\lambda$ is the threshold for $\hat{p}$.

Since the minimum of continuous functions is continuous, the only possible discontinuity for $\hat{p}_{i}$ comes when an agent $j$ switches sides. Note, however, that if $p\left(R_{i}^{n}\right) \rightarrow 0$, then $f\left(R_{i}^{n}\right) \rightarrow 0$ and so $\varphi_{j}\left(R^{n}\right) \rightarrow 0$ for all $j$. So $\varphi$ is continuous at $R$ if there is $i$ with $p\left(R_{i}\right)=0$. For all other $R \in \mathcal{R}^{N}, \hat{p}$ is clearly locally continuous, and $\varphi$ is the Uniform Rule composed with $\hat{p}$.

Thus, it remains to show strategy-proofness, and here we rely on the fact that $\left|f\left(R_{i}\right)\right| \geq\left|p\left(R_{i}\right)\right|$, so that $i$ 's report can only influence the derived peaks of agents asking for a larger trade. Thus, let us first show a property of the Uniform Rule.

Claim. Fix $\hat{p} \in \mathbb{R}^{N}$ and assume $\hat{p}_{i} \geq 0$. Let $\hat{q} \in \mathbb{R}^{N}$ have $\hat{q}_{j}=\hat{p}_{j}$ for $j$ with $\hat{p}_{j} \leq \hat{p}_{i}$. Then $U_{i}(\hat{q})=U_{i}(\hat{p})$.

Proof. We consider another method for calculating the Uniform rule. Let $\Omega=\Omega^{-}(\hat{p})=\Omega^{-}(\hat{q})$. For each $t \in[0, \Omega]$, the quantity $t$ is offered by an auctioneer to the agents on the positive side. At each $t$, the tentative allocation is $\xi_{i}^{t}=\hat{p}_{i}$ if $\hat{p}_{i}<t$ or $t$ otherwise. For $t$ sufficiently small, $\sum_{i \in N^{+}(\hat{p})} \xi_{i}^{t} \leq \min \left\{\Omega, \Omega^{+}(\hat{q})\right\}$ and there is a maximal value $T$ for which this holds. Then for each $i$ on the positive side, $U_{i}(\hat{p})=\xi_{i}^{T}$. Via this method, it is clear that, since the offer of the negative side remains constant, and the peaks less than $\hat{p}_{i}$ remain constant, that $i$ 's allocation is unchanged.

Denote by $\psi$ the collapsing Uniform Rule induced by $f$ and let $\xi=$ $\psi(R)$. Consider an agent with $p\left(R_{i}\right)>0$; the opposite case has a symmetric argument. Since $\left|f\left(R_{i}\right)\right| \geq\left|p\left(R_{i}\right)\right|$, derived peaks preserve the order of the primitive peaks. If $\xi_{i}=p\left(R_{i}\right)$ then there is clearly no profitable manipulation for $i$, so assume $\xi_{i} \neq p\left(R_{i}\right)$. Let $R_{i}^{\prime} \neq R_{i}$. Consider the change in the allocation that would result if the effect of the derived peaks on others were paused. That is, consider peak profile $\hat{p} \in \mathbb{R}^{N}$ such that $\hat{p}_{j}=\hat{p}_{j}(R)$ for $j \neq i$ and $\hat{p}_{i}=\hat{p}_{i}\left(R_{i}^{\prime}, R_{-i}\right)$. By construction,

$$
p\left(R_{i}\right) \geq \hat{p}_{i}(R) \geq \xi_{i}
$$


and since $\xi_{i} \neq p\left(R_{i}\right)$, one of these inequalities is strict. Suppose $p\left(R_{i}^{\prime}\right) \geq \xi_{i}$. If $p\left(R_{i}^{\prime}\right)<\hat{p}_{i}(R)$ it means that $\hat{p}_{i}(R)>\xi_{i}$ and so $\xi_{i}=\lambda$. Then, by the construction of derived peaks, we have $\hat{p}_{i}=p\left(R_{i}^{\prime}\right)$ and so, since $i$ is rationed at both $\hat{p}(R)$ and $\hat{p}, U(\hat{p})=U(\hat{p}(R))$. Otherwise, if $p\left(R_{i}^{\prime}\right) \geq \hat{p}_{i}(R)$, then $\hat{p}_{i}=\hat{p}_{i}(R)$ and again $U(\hat{p})=U(\hat{p}(R))$. In sum $p\left(R_{i}^{\prime}\right) \geq \xi_{i}$ implies $U(\hat{p})=\xi_{i}$.

Alternatively, $p\left(R_{i}^{\prime}\right)<\xi_{i}$ imples $p\left(R_{i}^{\prime}\right)<\hat{p}_{i}(R)$ and so $p\left(R_{i}^{\prime}\right) \leq \hat{p}_{i}<\xi_{i}$. Thus, $U_{i}(\hat{p})<\xi_{i}$. In particular, for $\hat{p}_{i} \geq 0, U_{i}(\hat{p})=\hat{p}_{i}$, and otherwise $i$ gets $\hat{p}_{i}$ until she is rationed at the other side of the market. In sum, $U_{i}(\hat{p}) \leq \xi_{i}$.

Now, in going from $\hat{p}$ to $\hat{q}=\hat{p}\left(R_{i}^{\prime}, R_{-i}\right)$, each $j$ with $\hat{p}_{j} \leq \hat{p}_{i}$ has $\hat{q}_{j}=\hat{p}_{j}$. Thus, by the claim and line 3.2 .

$$
\psi_{i}\left(R_{i}^{\prime}, R_{-i}\right)=U_{i}(\hat{q})=U_{i}(\hat{p}) \leq \xi_{i}=\psi_{i}\left(R_{i}\right) \leq p\left(R_{i}\right) .
$$

Single peakedness implies $\psi_{i}\left(R_{i}\right) R_{i} \psi_{i}\left(R_{i}^{\prime}, R_{-i}\right)$.

We have shown that rules satisfying our conditions belong to the family of B\&J rules; however, not all of these are continuous. In particular, the rule characterized in Barberà and Jackson (1995) induces large discontinuities as the trading regime switches from one line to another. By using a collapsing Uniform Rule on each trading line, this can be avoided. In particular, we may set $f\left(R_{i}\right)=\alpha p\left(R_{i}\right)$, for $\alpha>1$. The larger $\alpha$ is chosen, the more problems for which the induced continuous B\&J rule equals the original rule in their paper. In this sense, Barberà and Jackson's rule lies in the "closure" of the set of continuous B\&J rules, though we refrain from fully analyzing the topology of the space of rules.

A bossy, continuous BESJ rule. In their paper, Barberà and Jackson (1995) impose full non-bossiness. This, together with strategy-proofness implies group strategy-proofness, a fact that we do not have access to in this analysis. Some of our efforts might have been wasted if in fact our conditions imply full non-bossiness. We show now that they do not. 
For each $R_{i}$ and each $x \neq p\left(R_{i}\right)$, there is a unique number $r_{i}(x)$ such that $r_{i}(x) I_{i} x$. For each $R_{i} \in \mathcal{R}$, let

$$
f\left(R_{i}\right)= \begin{cases}r(-1) & p\left(R_{i}\right) \geq 0 \\ r(1) & \text { otherwise }\end{cases}
$$

The collapsing Uniform rule induced by $f$ is clearly bossy.

\section{Conclusion}

Somewhat novel in the literature, we have explored the consequences of informational restrictions, in a fairly abstract way, in market design. In doing so, we have arrived at a result that closely resembles the classic characterization of Barberà and Jackson (1995). Relative to their work, we deploy a weaker non-bossiness but a stronger continuity condition. Without full non-bossiness, we are unable to leverage the fact that this condition, coupled with strategy-proofness, implies weak group strategy-proofness. Thus we are able to more closely hone-in on the effect of individual incentives, rather than group incentives. However, by imposing our informational restriction, we arrive at a class of rules with basically the same structure. It should be emphasized, however, that we have only been able to do this in two dimensions, whereas Barberà and Jackson (1995) could work in arbitrary dimensions.

\section{Appendix A. Proof of Proposition 1}

For $x \in \mathbb{R}^{2}$, let $/ x /$ denote its angle with the horizontal axis. Given path $c \in \mathcal{C}$, let $c\left(\lambda^{*}\right)$ be the maximal point at which $c(\lambda) \in Z_{i}$. Thus, since $Z_{i}$ is rectangular, $L^{c}$ is indifferent between $c\left(\lambda^{*}\right)$ and the maximal point of $Z_{i}$. Let $\bar{c}$ be the path such that $\bar{c}(\lambda)=c(\lambda)$ for $c(\lambda) \in Z_{i}$ and $\bar{c}(\lambda) \in \llbracket c\left(\lambda^{*}\right), \max Z_{i} \rrbracket$ after. Let

$$
d^{1}\left(L^{c}, L^{c^{\prime}}\right)=\max _{x \in \bar{c}(\mathbb{R}), y \in \overline{c^{\prime}}(\mathbb{R})} \begin{cases}\left|x_{1}-y_{1}\right| & \left(x_{1}-x_{2}\right)\left(y_{1}-y_{2}\right) \geq 0 \\ \max \left\{\left|x_{1}-x_{2}\right|,\left|y_{1}-y_{2}\right|\right\} & \left(x_{1}-x_{2}\right)\left(y_{1}-y_{2}\right)<0\end{cases}
$$


and

$d^{2}\left(L^{c}, L^{c^{\prime}}\right)=\max _{x \in \bar{c}(\mathbb{R}), y \in \overline{c^{\prime}}(\mathbb{R})} \begin{cases}\left|x_{2}-y_{2}\right| & \left(x_{1}-x_{2}\right)\left(y_{1}-y_{2}\right) \geq 0 \\ \max \left\{\left|x_{1}-x_{2}\right|,\left|y_{1}-y_{2}\right|\right\} & \left(x_{1}-x_{2}\right)\left(y_{1}-y_{2}\right)<0 .\end{cases}$

The first function calculates the maximal horizontal difference one can find between the truncated versions of the paths associated with $L^{c}$ and $L^{c^{\prime}}$, where the distance is cut at the diagonal in the cases when it is crossed. The second function does the same with vertical distance. Finally, define $d^{*}\left(L^{c}, L^{c^{\prime}}\right)=\max \left\{d^{1}\left(L^{c}, L^{c^{\prime}}\right), d^{2}\left(L^{c}, L^{c^{\prime}}\right)\right\}$.

We first show the equivalence of $d$ and $d^{*}$. Fix two preferences $L^{c}$ and $L^{c^{\prime}}$ and their associated utility functions $u$ and $u^{\prime}$. Let $\lambda$ and $\lambda^{\prime}$ be parameters such that $u(c(\lambda))=u(x)$ and $u^{\prime}\left(c^{\prime}\left(\lambda^{\prime}\right)\right)=u^{\prime}(x)$. For now, suppress reference to the parameters. For generic $x$, the indifference sets of $L^{c}$ and $L^{c^{\prime}}$ meet $x$ orthogonally meaning that $/ c /, / x /$, and $/ c^{\prime} /$ will be strictly ordered, with $/ x /$ in the middle. Given generic $x$, assume

$$
\begin{aligned}
& c_{2}=x_{2}>x_{1}=c_{1}^{\prime} \\
& c_{2}>c_{1} \\
& c_{2}>c_{2}^{\prime} \\
& c_{1}<c_{1}^{\prime},
\end{aligned}
$$

where the first line simply fixes an order on the angles and simultaneously assumes $x$ is above the diagonal. The remaining lines are then consequences of the first, that $c$ also be above the diagonal and that $c$ and $c^{\prime}$ be antiordered in $\leq$. Clearly $u(x)=\sqrt{2} c_{2}$ and $u^{\prime}(x) \in\left\{\sqrt{2} c_{1}^{\prime}, \sqrt{2} c_{2}^{\prime}\right\}$, where the first case holds if $/ c^{\prime} \mid<\pi / 4$ and the second if $/ c^{\prime} / \geq \pi / 4$. Either way, the inequalities above give $u(x)>u^{\prime}(x)$.

If $x$ is in the interior of the consumption space, there is $y=x+\gamma(0,1)$ with $\gamma>0$. Since $x_{1}=c_{1}^{\prime}, y$ remains directly above $c^{\prime}$, so $u^{\prime}(y)=u^{\prime}(x)$. However, $y>c$, so $u(y)>u(x)$ and $u(y)-u^{\prime}(y)>u(x)-u^{\prime}(x)$. Thus, we can increase $\gamma$ until $y \in \bar{c}(\mathbb{R})$ and $u(y)-u^{\prime}(y)$ is increasing the entire way. Let $\mu$ have $u(c(\mu))=u(y)$ and note that $c_{2}(\mu)=y_{2}$, by construction. There 
are two cases. In the first $c^{\prime}(\lambda)$ is below the diagonal, so

$$
\begin{aligned}
u(y)-u^{\prime}(y) & =\sqrt{2}\left(c_{2}(\mu)-c_{1}^{\prime}(\lambda)\right) \\
& =\sqrt{2}\left(y_{2}-x_{1}\right) \\
& =\sqrt{2}\left(y_{2}-y_{1}\right),
\end{aligned}
$$

where we note that $y_{2}-y_{1}$ is the vertical distance from $y$ to the diagonal. When $c^{\prime}(\lambda)$ is above the diagonal,

$$
u(y)-u^{\prime}(y)=\sqrt{2}\left(y_{2}-c_{2}^{\prime}(\lambda)\right)
$$

in which case we have just multiplied the vertical distance between $y$ and $c^{\prime}(\lambda)$ by a constant. In sum, if $d\left(L^{c}, L^{c^{\prime}}\right)$ is found at $\left|u(x)-u^{\prime}(x)\right|$ with $x$ above the diagonal, then it is found at a point such as the above, where $y \in$ $\bar{c}(\mathbb{R})$ and $c(\lambda)$ are vertically oriented and we have $d\left(L^{c}, L^{c^{\prime}}\right)=\sqrt{2} d^{2}\left(L^{c}, L^{c^{\prime}}\right)$. It is then easy to see that, if $d\left(L^{c}, L^{c^{\prime}}\right)$ is maximized at a point below the diagonal, the entire argument reflects about the diagonal and yields $d\left(L^{c}, L^{c^{\prime}}\right)=\sqrt{2} d^{1}\left(L^{c}, L^{c^{\prime}}\right)$. In sum, $d\left(L^{c}, L^{c^{\prime}}\right)=\sqrt{2} d^{*}\left(L^{c}, L^{c^{\prime}}\right)$.

We now show that $\mathcal{C}$ has infinite Lebesgue covering dimension. Let $D^{*}$ be the path that first proceeds to point $(0,3)$ and afterward has the form $\lambda \mapsto(0,3)+\lambda(1,1)$. Let $D^{+}$and $D^{-}$, be the paths in $\mathcal{C}$ that form the upper and lower boundaries of the open ball $B_{1 / 2}\left(D^{*}\right)$, respectively. That is, $D^{+}$first proceeds to $(0,7 / 2)$ and then follows $\lambda \mapsto(0,7 / 2)+\lambda(1,1) ; D^{-}$is symmetric. Given $\varepsilon>0$, partition $\mathbb{R}^{2}$ in vertical pipes $\left\{P^{k}\right\}$ of width $\varepsilon$, so that the projection of $P^{k}$ on the horizontal axis is $] k \varepsilon,(k+1) \varepsilon$ [. For each odd $k$, let $c^{k}$ be the path that follows $D^{-}$at first, then within $P^{k}$, turns upward and meets $D^{+}$, and continues to follow $D^{+}$thereafter. Thus, $D^{*} \in B_{1}\left(c^{k}\right)$. However, for each distinct pair $c^{k}$ and $c^{j}$, letting $j>k$, we have a vertical distance of 1 between $c^{k}$ and $c^{j}$ within $P^{k+1}$. Thus, $d^{*}\left(c^{k}, c^{j}\right) \geq 1$.

The family $\left\{B_{1}\left(c^{k}\right): k \in 1, \ldots, K\right\} \cup\left\{B_{1 / 2}\left(c^{\prime}\right): c^{\prime} \notin B_{1}\left(c^{k}\right), k \in 1, \ldots, K\right\}$ is, by construction, a cover of $\mathcal{C}$. Let $\left\{B^{i}\right\}_{i \in I}$ be a refinement, and suppose there is $B_{1}\left(c^{k}\right)$ that does not have any member $B^{i}$ as a subset. Then for each $B^{i}$, either $B^{i} \subseteq B_{1}\left(c^{j}\right)$ for $j \neq k$, or $B^{i} \subseteq B_{1 / 2}\left(c^{\prime}\right)$ for $c^{\prime} \notin B_{1}\left(c^{j}\right)$ for any $j$. 
In the first case, as shown above, $c^{k} \notin B^{i}$. In the second, $d^{*}\left(c^{k}, c^{\prime}\right) \geq 1$ and so $c^{k} \notin B_{1 / 2}\left(c^{\prime}\right)$. Thus, $c^{k}$ is not covered by the refinement, and it follows that any refinement covering $\mathcal{C}$ must contain subsets of every $B_{1}\left(c^{k}\right)$. As noted above, each of these contains $D^{*}$, and we can make arbitrarily many of them by choosing $\varepsilon$ sufficiently small. Thus, $\mathcal{C}$ has infinite Lebesgue covering dimension.

\section{Appendix B. Proof of Theorem 1}

Some notation: For each profile $R \in \mathcal{R}^{N}$ and each agent $i$, let $A_{i}\left(R_{-i}\right)=$ $\varphi_{i}\left(\mathcal{R}, R_{-i}\right)$. This is referred to as agent $i$ 's attainable set at profile $R$. Anonymity implies that for each $i, j \in N, A_{i}=A_{j}=$ : $A$. For each pair $\{x, y\} \subset \mathbb{R}^{2}$, let

$$
\begin{aligned}
& \llbracket x, y \rrbracket:=\left\{z \in \mathbb{R}^{2}: z=\theta x+(1-\theta) y, 0 \leq \theta \leq 1\right\} \\
& \llbracket x, y \llbracket:=\left\{z \in \mathbb{R}^{2}: z=\theta x+(1-\theta) y, 0 \leq \theta<1\right\} \\
& \rrbracket x, y \llbracket:=\left\{z \in \mathbb{R}^{2}: z=\theta x+(1-\theta) y, 0<\theta<1\right\} .
\end{aligned}
$$

Given a vector $x \in \mathbb{R}_{++}^{2}$, denote by $L^{x}$ the Leontief preference given by a terminal path of the form $\lambda \mapsto \lambda x$. These preferences are obviously generated by the set $\Theta=\left\{\theta \in \mathbb{R}_{++}^{2}:\|\theta\|=1\right\}$. A linear preference relation $R_{i}$ is such that there exists $r \in \mathbb{R}^{2}$ making the inner product $\langle\cdot, r\rangle$ a representation of $R_{i}$. Note that linear preference relations are not in our preference domain; they are a useful tool.

B.1. Without Simplicity. For this subsection, we assume $\varphi$ satisfies all the properties except simplicity.

This first lemma is an obvious consequence of strategy-proofness, and so we omit its proof.

Lemma 1. For each agent $i \in N$, and each $x \in A\left(R_{-i}\right), \varphi_{i}(R) R_{i} x$.

A set $B \subset \mathbb{R}^{2}$ is an anti-chain if each pair in the set is not comparable by $\leq$, that is, if for each $\{x, y\} \subseteq B, x \neq y$ implies $\neg(x \geq y)$ and $\neg(y \geq x)$.

Lemma 2. For each $R \in \mathcal{R}^{N}$, and each $i \in N, A\left(R_{-i}\right)$ is an anti-chain. 
Proof. Assume that there are $x$ and $y$ in $A\left(R_{-i}\right)$ such that $y \gtrless x$. Let $R \in \mathcal{R}^{N}$ satisfy $\varphi_{i}(R)=x$ and $R_{i}^{\prime} \in \mathcal{R}$ satisfy $\varphi_{i}\left(R_{i}^{\prime}, R_{-i}\right)=y$. Since $\varphi$ is strategy-proof and preferences are monotone, $y I_{i} x$ and $R_{i}$ must be a Leontief relation. Let $\left(R_{i}^{\nu}\right)_{\nu \in \mathbb{N}}$ be a sequence of strictly increasing preference relations converging to $R_{i}$. Then for each $\nu, \varphi_{i}\left(R_{i}^{\nu}, R_{-i}\right)=x$, contradicting continuity.

Corollary 1. For each $R \in \mathcal{R}^{N}$ and each $i \in N$, letting $x=\varphi_{i}(R)$ and $c \in \mathcal{C}$ cross $x$ at some point, it follows that $\varphi_{i}\left(L^{c}, R_{-i}\right)=\varphi_{i}(R)$.

Proof. Note that a Leontief preference can be indifferent between two bundles $x$ and $y$ only if they are $\geq$-ordered in some way. Thus, the choice of any $L^{c}$ from any antichain set will be a singleton and an element of the path $c$.

Lemma 3. For each $R \in \mathcal{R}^{N}$, and each $i \in N, A\left(R_{-i}\right)$ is compact-valued and connected-valued.

Proof. The set $\Theta$ is connected and compact. Let $\mathcal{L}^{\Theta}:=\left\{L^{x}: x \in \Theta\right\}$. It is clear that the mapping $x \mapsto L^{x}$ is continuous and therefore $\mathcal{L}^{\Theta}$ is connected and compact. Thus, since $\varphi$ is continuous, $\varphi_{i}\left(\mathcal{L}^{\Theta}, R_{-i}\right)$ is also connected and compact.

Let $x \in A\left(R_{-i}\right)$. Since $A\left(R_{-i}\right)$ is an anti-chain, $\varphi\left(L_{i}^{x}, R_{-i}\right)=x$ and it follows that $\varphi\left(\mathcal{L}^{\Theta}, R_{-i}\right)=A\left(R_{-i}\right)$.

An anti-chain $A \subset \mathbb{R}^{2}$ bends away from the origin if for each pair $x, y \in$ $A$, each $\gamma \in \llbracket x, y \rrbracket$ and each $z \in A$, if $\gamma \geq z$ then $\gamma=z$. Equivalently, $A$ is the upper envelope, in $\leq$, of a convex set. The following lemma is proven as Lemma 1 in Sprumont (1995).

Lemma 4. For each profile $R \in \mathcal{R}^{N}$ and for each agent $i \in N, A\left(R_{-i}\right)$ bends away from the origin.

B.2. Bringing in Simplicity. Henceforth assume that $\varphi$ satisfies all the properties in Theorem 1 .

Lemma 5. If $\sigma$ encodes $\varphi$ then the induced mapping $\varphi^{*}$ is continuous. 
Proof. Since $\mathcal{M} \hookrightarrow \mathbb{R}^{k}$ for some $k \in \mathbb{N}$, its topology can be described via sequences, rather than the more general nets. Suppose there is an element $m \in \mathcal{M}$ such that $\varphi^{*}$ is not continuous at $m$. Thus there is convergent $m^{n} \rightarrow m$ such that $\lim \varphi^{*}\left(m^{n}\right) \neq \varphi^{*}(m)$. Since $\left\{m^{n}\right\}_{n \in \mathbb{N}} \cup\{m\}$ is closed, so is its pre-image under $\sigma$ and it is therefore also compact. For each $n$ there is $R^{n} \in \mathcal{R}^{N}$ with $\sigma\left(R^{n}\right)=m^{n}$. By compactness, since $\mathcal{R}$ is metrizable, there is a convergent subsequence $R^{s(n)}$, with limit $R$, and $\lim \sigma\left(R^{s(n)}\right)=m=\sigma(R)$. We conclude that

$$
\begin{aligned}
\lim \left(\varphi^{*} \circ \sigma\right)\left(R^{n}\right) & =\lim \varphi^{*}\left(m^{n}\right) \\
& \neq \varphi^{*}(m) \\
& =\left(\varphi^{*} \circ \sigma\right)(R),
\end{aligned}
$$

contradicting the continuity of $\varphi$, as $\varphi^{*} \circ \sigma=\varphi$.

Lemma 6. $\varphi, \sigma$, and $\varphi^{*}$ are closed mappings.

Proof. Let $A \subset \mathcal{R}$ be closed. As a closed subset of a compact space, $A$ is compact. Since $\varphi$ and $\sigma$ are continuous, $\varphi(A)$ and $\sigma(A)$ are compact, and thus closed. If $A \subseteq \mathcal{M}$ is closed, then since $\sigma$ is continuous and $\varphi$ is closed, $\varphi^{*}(A)=\left(\varphi \circ \sigma^{-1}\right)(A)$ is closed. So $\varphi^{*}$ is a closed map.

Since $A\left(R_{-i}\right)$ is an antichain, each of its elements may be uniquely identified in polar coordinates by an angle in $\left[0, \frac{\pi}{2}\right]$ and a distance. It will be more convenient for us, however, to identify $\left[0, \frac{\pi}{2}\right]$ with $\Theta$, so we interchangeably view elements of $\Theta$ as angles and vectors.. To avoid confusion, we write $\theta^{\prime} \succ \theta$ when $\sin ^{-1}\left(\theta_{2}^{\prime}\right)>\sin ^{-1}\left(\theta_{2}\right)$. Thus, $\succ$ linearly orders $\Theta$ anti-clockwise. We will therefore speak of intervals $I \subseteq \Theta$, with upper and lower bounds according to $\succ$, while also identifying arbitrary vectors in $\mathbb{R}_{+}^{2}$ via $r \theta$ for $r \in \mathbb{R}_{+}$. Conversely, given $x \in \mathbb{R}^{2}$, let $/ x / \in \Theta$ denote it's angle. Note that $/ \cdot /$ allows us to extend the order $\succ$ to $\mathbb{R}_{+}^{2}$ as follows: if $\{x, y\}$ is an antichain, then $x \succ y$ if and only if $|x|>|y|$. 
For each $R_{-i}$, there are a set $K_{i}\left(R_{-i}\right) \subseteq \Theta$ and an injective function $\alpha_{i}\left(\cdot ; R_{-i}\right): K_{i}\left(R_{-i}\right) \rightarrow A\left(R_{-i}\right)$. As above, we will typically omit the subscript on $K$. Moreover, $\alpha_{i}$ is (jointly) continuous in all arguments and $K$ is a closed interval. Let

$$
\begin{aligned}
& \alpha_{i}^{r}\left(R_{-i}\right)=\alpha_{i}\left(\min K\left(R_{-i}\right) ; R_{-i}\right) \\
& \alpha_{i}^{l}\left(R_{-i}\right)=\alpha_{i}\left(\max K\left(R_{-i}\right) ; R_{-i}\right) .
\end{aligned}
$$

Thus, the set $A\left(R_{-i}\right)$ is path. Each point on the path is the image of some $R_{i} \in \mathcal{R}$ under $\varphi_{i}\left(\cdot, R_{-i}\right)$. Since $\varphi$ is continuous, the paths $\left\{A\left(R_{-i}\right): R_{-i} \in \mathcal{R}^{N \backslash i}\right\}$ continuously deform into each other. That is, given a path $\nu \in[0,1] \mapsto R_{-i}^{\nu}$, $\left\{A\left(R_{-i}^{\nu}\right)\right\}_{\nu \in \mathbb{R}}$ is a homotopy. Consider a path $\nu \in[0,1] \mapsto R_{-i}^{\nu} \in \mathcal{R}^{N \backslash\{i\}}$. By Corollary 1 .

$$
\left\{A\left(R^{\nu}\right)\right\}_{\nu \in[0,1]}=\left\{\varphi_{i}\left(L^{c}, R_{-i}^{\nu}\right)\right\}_{(c, \nu) \in \mathcal{C} \times[0,1]} .
$$

Proposition 3. Let $R_{-j}^{\nu}$ be a path in $\mathcal{R}^{N \backslash j}$. Let $\{y, x\} \subseteq A\left(R_{-j}^{0}\right)$ be an antichain and let $\mathcal{N}$ be the set of $\nu$ such that $\{y, x\} \subseteq A\left(R_{-j}^{\nu}\right)$. Let $\nu^{*}=$ $\inf ([0,1] \backslash \mathcal{N})]^{6}$ Then if $\nu^{*}$ is finite, it is a member of $\mathcal{N}$. Letting $I \subseteq \Theta$ be the interval with endpoints $/ y /$ and $/ x /$, we have, for each $\nu \leq \nu^{*}$,

$$
\left.\alpha_{j}\left(\cdot ; R_{-j}^{\nu}\right)\right|_{I}=\left.\alpha_{j}\left(\cdot ; R_{-j}^{0}\right)\right|_{I}
$$

Finally if $\nu^{*}$ is finite, then one of the endpoints of $I$ is also an endpoint of $K\left(R_{-j}^{\nu^{*}}\right)$.

Proof. For notational simplicity, since we are considering only the path $R_{-i}^{\nu}$, we replace $R_{-i}^{\nu}$ by $\nu$ as an argument in the $A, K$, and $\alpha$ functions.

Note that the angles $I \subseteq \Theta$ identify a closed cone $\bar{V} \in \mathbb{R}^{2}$, with nonempty interior $V$. For each $\nu \in \mathcal{N}$, since $A_{i}(\nu)$ is an antichain, $C\left(L^{x}, A_{i}(\nu)\right)=$ $\{x\}$. By strategy-proofness, $\varphi_{i}\left(L^{x}, R_{-i}^{\nu}\right)=x$. Similarly, $\varphi_{i}\left(L^{y}, R_{-i}^{\nu}\right)=y$. Closedness of $\mathcal{N}$ then follows from continuity, which then yields $\nu^{*} \in \mathcal{N}$.

$\overline{{ }^{6} \text { We adopt the }}$ usual convention that $\inf \emptyset=\infty$. 
Moreover, for each $\nu \in \mathcal{N},\{/ y /, / x /\} \subseteq K_{i}(\nu)$ and so it follows since attainable sets are connected that $I \subseteq K_{i}(\nu)$. Then for each $\theta \in I$ and each $\nu \in \mathcal{N}, \alpha_{i}(\theta ; \nu)$ is defined and, by definition, equals $\left\|\alpha_{i}(\theta ; \nu)\right\| \theta$. As $A_{i}(\nu)$ is an antichain, again we have $C\left(L^{\theta}, A_{i}(\nu)\right)=\left\{\alpha_{i}(\theta ; \nu)\right\}$. Thus, by strategy-proofness,

$$
\forall \nu \in \mathcal{N}, \forall \theta \in I, \varphi_{i}\left(L^{\theta}, R_{-i}^{\nu}\right)=\alpha_{i}(\theta ; \nu)
$$

Let $\nu<\nu^{*}, \theta \in I$, and

$$
S:=\left\{x \in \mathbb{R}^{2}: \alpha_{i}(\theta ; 0)<x<\alpha_{i}(\theta ; \nu)\right\}
$$

Suppose $S$ is non-empty. Let $r \theta^{\prime} \in S \cap V$. By line B.1.

$$
\varphi_{i}\left(L^{\theta^{\prime}}, R_{-i}^{0}\right)=\alpha_{i}\left(\theta^{\prime} ; 0\right)=\left\|\alpha_{i}\left(\theta^{\prime} ; 0\right)\right\| \theta^{\prime}<r \theta^{\prime}<\alpha_{i}(\theta ; \nu)
$$

where the first inequality is because otherwise $\alpha_{i}\left(\theta^{\prime} ; 0\right) \geq \alpha_{i}(\theta ; 0)$, contradicting that $A_{i}(0)$ is an antichain. Since $\alpha_{i}\left(\theta^{\prime} ; \nu\right)$ is $\succ$-ordered with $\alpha_{i}(\theta ; \nu)$, there is $\mu<\nu$ with $\left\|\alpha_{i}\left(\theta^{\prime} ; \mu\right)\right\|>r$. By the intermediate value theorem then, there is $\mu^{\prime}$ with $\left\|\alpha_{i}\left(\theta^{\prime} ; \mu^{\prime}\right)\right\|=r$ and so $\varphi_{i}\left(L^{\theta^{\prime}}, R_{-i}^{\mu^{\prime}}\right)=r \theta$. In sum, for each $x \in S \cap V$, there are $\theta^{\prime}=/ x / \in I$ and $\nu^{\prime} \in\left[0, \nu^{*}\right]$ with $\varphi_{i}\left(L^{\theta^{\prime}}, R_{-i}^{\nu^{\prime}}\right)=x$.

Since $S \cap V$ is open it contains open $U$ with the following shape: there are $x^{*}$ and $\bar{\lambda}>\left\|x^{*}\right\|=: \underline{\lambda}$ such that

$$
U=\left\{y: y>x^{*},\|y\|<\bar{\lambda}\right\}
$$

That is, $U$ a small wedge. Since the paths in $\mathcal{C}$ are increasing, we may parameterize each of them so that $\|c(\lambda)\|=\lambda$. Let $\mathcal{C}^{U}$ be the subset of terminal monotone paths in $\mathbb{R}^{2}$ such that each $c \in \mathcal{C}^{U}$ has $c(\underline{\lambda})=x^{*}$. It follows that $c$ meets the boundary of $U$ at $c(\bar{\lambda})$. Define equivalence relation $\sim$ on $\mathcal{C}^{U}$ such that $c \sim c^{\prime}$ if and only if $c([0,1]) \cap U=c^{\prime}([0,1]) \cap U$. Clearly $\mathcal{C}^{U} / \sim$ is homeomorphic to $\mathcal{C}$. We show that $\mathcal{C}^{U} / \sim \hookrightarrow \mathcal{M}$, arriving at a contradiction, since $\mathcal{C}$ cannot be embedded in any finite dimensional Euclidean space. 
We first show that $\mathcal{C}^{U} / \sim$ maps injectively to $\mathcal{M}$. Suppose paths $c$ and $c^{\prime}$ are not equivalent. Then there is $\lambda \in[\underline{\lambda}, \bar{\lambda}]$ such that that $c(\lambda) \neq c^{\prime}(\lambda)$. Since both are in $U \subseteq S \cap V, \vartheta=/ c(\lambda) /$ and $\vartheta^{\prime}=/ c^{\prime}(\lambda) /$ are in int $I$. Moreover, there is $\mu \in\left[0, \nu^{*}\right]$ such that $\varphi_{i}\left(L^{\vartheta}, R_{-i}^{\mu}\right)=c(\lambda)=\alpha_{i}(\vartheta ; \mu)$. By strategy-proofness $\varphi_{i}\left(L^{c}, R_{-i}^{\mu}\right)=\varphi_{i}\left(L^{\vartheta}, R_{-i}^{\mu}\right)$. Since $\vartheta^{\prime}$ is interior to $I \subseteq K_{i}(\mu), \varphi_{i}\left(L^{c}, R_{-i}^{\mu}\right) \in C\left(L^{c^{\prime}}, A\left(R_{-i}^{\mu}\right)\right)$ if and only if $\varphi_{i}\left(L^{c}, R_{-i}^{\mu}\right)=$ $c(\lambda)$ is the intersection of $c^{\prime}$ and $A_{i}(\mu)$, implying by our parameterization that $c^{\prime}(\lambda)=c(\lambda)$. Thus, $\varphi_{i}\left(L^{c}, R_{-i}^{\mu}\right) \neq \varphi_{i}\left(L^{c^{\prime}}, R_{-i}^{\mu}\right)$, and moreover this inequality is true on an open neighborhood of $\mu$. Thus, there is an injection $\xi: \mathcal{C}^{U} / \sim \rightarrow \subseteq \mathcal{M}_{i}$.

We wish to show that $\xi$ is a homeomorphism. Let $\mathcal{L}^{U}$ be the set of Leontief preferences induced by paths $c \in \mathcal{C}^{U}$. We use the diagram below, where $i d$ is the identity mapping:

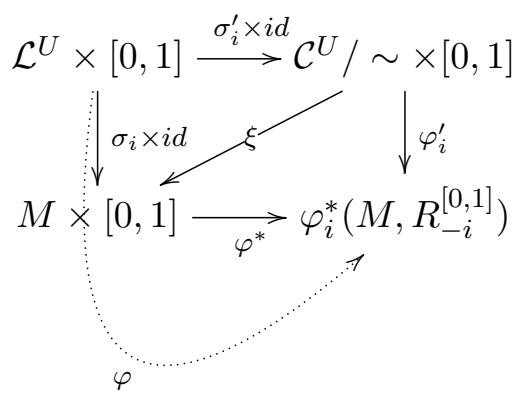

That is, let $\sigma_{i}^{\prime}$ be the obvious mapping from Leontief preference relations to paths in $\mathcal{C}^{U} / \sim$, which is clearly a homeomorphism. Let $\varphi_{i}^{\prime}$ then be the mapping that then gives allocations from reports of the form $(c, \nu)$. That is, $\varphi_{i}^{\prime}$ is essentially $\varphi_{i}$ restricted to $\mathcal{L}^{U} \times R^{[0,1]}$. Observe then that $\mathcal{C}^{U} / \sim$ maps continuously into $\varphi_{i}^{*}\left(M, R_{-i}^{[0,1]}\right)$ via $\varphi_{i}^{\prime}$. Thus, if $A \subseteq M$ is closed, $\varphi^{\prime-1}\left(\varphi^{*}(A)\right)$ is closed by the closedness of $\varphi^{*}$ and continuity of $\varphi^{\prime}$. This shows that $\xi$ is continuous. By Lemma 6, $\sigma_{i}$ is closed, so if $A \subseteq \mathcal{C}^{U} / \sim$ is closed, then $\left(\sigma \circ \sigma^{\prime-1}\right)(A)$ is closed. This shows that $\xi$ is closed. In sum, $\xi$ is a continuous, closed bijection, and therefore a homeomorphism. Thus we have that $\mathcal{C}^{U} / \sim$ is homeomorphic to $M \subseteq \mathcal{M}_{i}$, a contradiction, as $\mathcal{C}$ cannot be embedded in any finite dimensional Euclidean space. 
Conclude that $S$ is empty and so $\alpha_{i}(\theta ; \nu)=\alpha_{i}(\theta ; 0)$. Since $\nu$ was an arbitrary parameter not greater than $\nu^{*}$, and $\theta$ was an arbitrary member of int $I$, we are done.

Finally, if neither endpoint of $I$ is an endpoint of $K\left(\nu^{*}\right)$, then $I \subseteq$ $\operatorname{int}\left(K\left(\nu^{*}\right)\right)$ and we may invoke the above arguments again to conclude that $\{y, x\} \subseteq A_{j}(\nu)$ for some $\nu>\nu^{*}$, a contradiction.

Say that $x \in A\left(R_{-i}\right)$ is a (relative) interior point if $/ x / \in \operatorname{int}\left(K\left(R_{-i}\right)\right)$. The following lemma then demonstrates how the attainable set is traced by its endpoints.

Lemma 7. Let $R_{-i}^{\nu}$ be a path in $\mathcal{R}^{N \backslash i}$. Suppose that $x \notin A\left(R_{-i}^{\nu}\right)$ but is an interior element of $A\left(R_{-i}^{\nu^{\prime}}\right)$ for $\nu^{\prime}>\nu$. Then there is $\left.\nu^{*} \in\right] \nu, \nu^{\prime}[$ such that $x \in\left\{\alpha_{i}^{l}\left(R_{-i}^{\nu^{*}}\right), \alpha_{i}^{r}\left(R_{-i}^{\nu^{*}}\right)\right\}$. Symmetrically, if $x$ is an interior point of $A\left(R_{-i}^{\nu}\right)$ and does not belong to $A\left(R_{-i}^{\nu^{\prime}}\right)$ for some $\nu^{\prime}>\nu$, then there is $\left.\nu^{*} \in\right] \nu, \nu^{\prime}[$ such that $x \in\left\{\alpha_{i}^{l}\left(R_{-i}^{\nu^{*}}\right), \alpha_{i}^{r}\left(R_{-i}^{\nu^{*}}\right)\right\}$.

Proof. Note that the two claims of the lemma are actually equivalent: if one is true then it implies the other by running the path $R^{\nu}$ in reverse. Thus, we shall prove the second claim as it allows us to more directly apply the previous lemma.

Since $x$ is interior to $A\left(R_{-i}^{\nu}\right)$, the attainable set is non-trivial, so there is $y^{1} \in A\left(R_{-i}^{\nu}\right)$, interior, such that $\left\{y^{1}, x\right\}$ is an antichain. Let $\mathcal{N}^{1} \subseteq[\nu, 1]$ be the set of parameters $\mu$, at least $\nu$, with $\left\{y^{1}, x\right\} \subseteq A\left(R_{-i}^{\mu}\right)$. Let $\nu^{1}$ correspond to $\nu^{*}$ in Proposition 3 . Since $x$ and $y$ are interior at $\nu$, and $x$ leaves the attainable set at some point, $\nu<\nu^{1}<1$. The proposition further implies that $\left\{/ y^{1} /, / x /\right\}$ contains an endpoint of $K\left(\nu^{1}\right)$. Letting $I^{1}$ be the interval with endpoints $/ y^{1} /$ and $/ x /$, we have also that $\left.\alpha_{i}(\cdot ; \nu)\right|_{I^{1}}$ is constant in $\nu$ up to and including $\nu^{1}$. Thus, $\left\{\alpha_{i}^{l}\left(\nu^{1}\right), \alpha_{i}^{r}\left(\nu^{1}\right)\right\} \cap\left\{x, y^{1}\right\} \neq \emptyset$. If the intersection contains $x$ we are done. If not, then there is $y^{2}$ interior to $A_{i}\left(\nu^{1}\right)$ such that $\left\{y^{2}, x\right\}$ forms an antichain. In particular, we may choose $y^{2}$ so that $\left|/ y^{2} /-\right| x /\left|<\frac{1}{2}\right| / y^{1} /-/ x / \mid$. Thus, if repetition of the above argument results in an infinite sequence $y^{n}$, then $y^{n} \rightarrow x$ and the argument then follows from continuity of the $\alpha^{l}$ and $\alpha^{r}$ functions. 
For each $k \in\{1, \ldots,|N|-1\}$, let $L^{k}:=\left(L_{1}^{(1,0)}, \ldots, L_{k}^{(1,0)}, L_{k+1}^{(0,1)}, \ldots, L_{|N|}^{(0,1)}\right)$. By anonymity, for each $k$, there are $x^{k}$ and $y^{k} \in \mathbb{R}^{2}$ such that for each $i \in N$, if $L_{i}^{k}=L^{(1,0)}$ then $\varphi_{i}\left(L^{k}\right)=x^{k}$ and otherwise, $\varphi_{i}\left(L^{k}\right)=y^{k}$. Let $q^{k}$ be a normal vector to line segment $\llbracket x^{k}, y^{k} \rrbracket$. Feasibility implies $\omega \in \llbracket x^{k}, y^{k} \rrbracket$.

Lemma 8. For each $k$ such that $x^{k} \neq \omega$, and each $i \leq k, \llbracket \omega, x^{k} \rrbracket \subseteq A\left(L_{-i}^{k}\right)$. Symmetrically, for $i>k, \llbracket y^{k}, \omega \rrbracket \subseteq A\left(L_{-i}^{k}\right)$.

Proof. Let $\theta^{\omega}=/ \omega /$. Construct the path $\nu \in\left[\theta^{\omega},(0,1)\right] \subseteq \Theta \mapsto R^{\nu}$ by setting

$$
R_{i}^{\nu}= \begin{cases}L^{(1,0)} & i \leq k \\ L^{\nu} & i>k\end{cases}
$$

By anonymity then, each side of $k$ has a common bundle under $\varphi$. In particular, for each $i \leq k$, strategy-proofness and the anti-chain property of attainable sets imply $\varphi_{i}\left(R^{\nu}\right)=\alpha_{1}^{r}(\nu)$. For the opposite side, each $i>k$ has $\varphi_{i}\left(R^{\nu}\right)=\varphi_{\bar{n}}\left(R^{\nu}\right)$, where we let $\bar{n}=|N|$. By feasibility,

$$
\varphi_{\bar{n}}\left(R^{\nu}\right)=\frac{\bar{n} \omega-k \alpha_{1}^{r}(\nu)}{\bar{n}-k}
$$

so the allocation of one side is the weighted reflection through $\omega$ of the allocation of the other. The approach of our argument is then to use the fact that $\alpha_{1}^{r}$ traces the (common) attainable set of the $i \leq k$ agents, which means it must move in arcs bending away from the origin. This then implies that $\varphi_{\bar{n}}\left(R^{\nu}\right)$ must move in arcs bending toward the origin. Thus if we can show that $\varphi_{\bar{n}}\left(R^{\nu}\right)$ also traces an attainable set then we must have them both linear.

By voluntary participation, $\varphi_{i}\left(R^{0}\right)=\omega$ for all $\iota$. Assume $\varphi_{1}\left(R^{\nu}\right) \neq \omega$. Thus by feasibility and voluntary participation, $\varphi_{\bar{n}}\left(R^{\nu}\right) \succ \omega \succ \varphi_{1}\left(R^{\nu}\right)$. Further, since attainable sets are path connected, there is $x \in A_{1}(\nu)$, interior, with $\omega \succ x$. Note that, since $A_{1}$ is antichain valued, if there were $\nu^{\prime}$ with $\alpha_{1}^{l}\left(\nu^{\prime}\right)=x$ then each $x^{\prime} \in A_{1}\left(\nu^{\prime}\right)$ would have $\omega \succ x \succ x^{\prime}$, in violation of voluntary participation. Thus, $\alpha_{1}^{l}$ cannot touch $x$ on the path $R^{\nu}$. It follows by Lemma 7, that there is at least one $\nu^{\prime}<\nu$ such that $\alpha_{1}^{r}\left(\nu^{\prime}\right)=x$. The 
set of such $\nu^{\prime}$ that is less than $\nu$ is closed, and it has a maximal element $\nu^{*}$. Thus, again by Lemma 7, $x \in A_{1}(\mu)$ for all $\mu \in\left[\nu^{*}, \nu\right]$ and is interior to the attainable set for all these except $\nu^{*}$.

For each $\mu \in\left[\nu^{*}, \nu\right]$, there is $z \in A_{1}(\mu)$ with $z \geq \omega$. Let $q \in \mathbb{R}_{+}^{2}$ be normal to $\llbracket \omega, x \rrbracket$ and suppose $q \alpha_{1}^{r}(\mu)>q x$. Since $x \in A_{1}(\mu)$, we have $x \succ \alpha_{1}^{r}(\mu)$. Since $\omega \succ x, x \neq z$ and so $z \succ x$, as $z$ and $x$ must be anti-ordered in some way. In sum, $z \succ x \succ \alpha_{1}^{r}(\mu)$. There is $x^{\prime} \in \llbracket z, \alpha_{1}^{r}(\mu) \rrbracket$ with $/ x^{\prime} /=/ x /$. Conclude that

$$
\begin{aligned}
q x^{\prime} & =q\left(\lambda z+(1-\lambda) \alpha_{1}^{r}(\mu)\right) \\
& >\lambda q \omega+(1-\lambda) q x \\
& =\lambda q x+(1-\lambda) q x \\
& =q x,
\end{aligned}
$$

in violation of Lemma 4. Therefore, $q \alpha_{1}^{r}(\mu) \leq q x$. Note that $x=\varphi_{1}\left(R^{\nu^{*}}\right)$, and let $y=\varphi_{\bar{n}}\left(R^{\nu^{*}}\right)$. It follows, by also considering feasibility, that for each $\left.\mu \in] \nu^{*}, \nu\right]$,

$$
\begin{gathered}
q \varphi_{\bar{n}}\left(R^{\mu}\right) \geq q y=q \omega=q x \geq q \alpha_{1}^{r}(\mu)=q \varphi_{1}\left(R^{\mu}\right), \\
\varphi_{\bar{n}}\left(R^{\mu}\right) \succ y \succ \omega \succ x \succ \alpha_{1}^{r}(\mu)=\varphi_{1}\left(R^{\mu}\right) .
\end{gathered}
$$

If there is $\left.\mu \in] \nu^{*}, \nu\right]$ with $y \in A_{\bar{n}}(\mu)$, then line B.3 implies it is interior. Thus, by Lemma 7 and the fact that line B.3 holds for arbitrary $\left.\mu \in] \nu^{*}, \nu\right]$, $y \in A_{\bar{n}}(\nu)$. Then Lemma 4 applied to line B.2 makes the inequalities equalities and yields $\llbracket \varphi_{\bar{n}}\left(R^{\nu}\right), \omega \rrbracket \subseteq A_{\bar{n}}(\nu)$. Since $\nu$ is arbitrary, this yields the lemma.

Assume, therefore, that for each $\left.\mu \in] \nu^{*}, \nu\right], y \notin A_{\bar{n}}(\nu)$. Let $\mu^{n} \searrow \nu^{*}$. For each $n$, there is $z^{n} \in A_{\bar{n}}\left(\mu^{n}\right)$ with $z^{n} \geq \omega$. Suppose that each $n$ has $/ z^{n} / \geq$ $/ y /$. Note that if $z^{n} \succ \varphi_{\bar{n}}\left(R^{\mu^{n}}\right)$, then by line B.3. $z^{n} \succ \omega$, a contradiction. Therefore, since $\varphi_{\bar{n}}\left(R^{\mu^{n}}\right)$ must be $\succ$-ordered with $z^{n}, \varphi_{\bar{n}}\left(R^{\mu^{n}}\right) \succ z^{n}$. In sum,

$$
/ \varphi_{\bar{n}}\left(R^{\mu^{n}}\right) />/ z^{n} / \geq / y / .
$$


Since $\varphi_{\bar{n}}\left(R^{\mu^{n}}\right) \rightarrow y$, and $/ \cdot /$ is continuous, $\lim / z^{n} /=/ y /$, a contradiction since $y \succ \omega$ and the set of points $\succ$-ordered with $\omega$ is open. Thus, we may choose $\left.\mu \in] \nu^{*}, \nu\right]$ such that there is $z \in A_{\bar{n}}(\mu)$ with $z \geq \omega$ and

$$
\mid \varphi_{\bar{n}}\left(R^{\mu}\right) />/ y />/ z /
$$

Moreover, since $A_{\bar{n}}(\mu)$ bends away from the origin, for each $\theta \in\left[/ z /, / \varphi_{\bar{n}}\left(R^{\mu}\right) /\right]$, $\alpha_{\bar{n}}(\theta ; \mu)$ dominates some element of $\llbracket \varphi_{\bar{n}}\left(R^{\mu}\right), z \rrbracket$. Thus, there is $\lambda \in[0,1]$ with

$$
\begin{array}{rlr}
q \alpha_{\bar{n}}(/ y / ; \mu) & \geq q\left(\lambda \varphi_{\bar{n}}\left(R^{\mu}\right)+(1-\lambda) z\right) & \\
& \geq \lambda q y+(1-\lambda) q \omega & \text { recalling line B.2 } \\
& =\lambda q y+(1-\lambda) q y=q y, &
\end{array}
$$

Therefore, $y^{\prime}:=\alpha_{\bar{n}}(/ y / ; \mu)>y$, and $y^{\prime}$ is interior to $A_{\bar{n}}(\mu)$. Since $y^{\prime}$ and $y$ cannot be together in an attainable set, while $y \in A_{\bar{n}}\left(\nu^{*}\right)$, Lemma 7 implies there is some $\left.\mu^{\prime} \in\right] \nu^{*}, \mu\left[\right.$ with $\alpha_{\bar{n}}^{l}\left(\mu^{\prime}\right)=y^{\prime}$. As above we can choose $\mu^{\prime}$ so that $y^{\prime}$ is interior to $A_{\bar{n}}(\gamma)$ for each $\left.\gamma \in\right] \mu^{\prime}, \mu[$.

Recall that the path $R^{\nu}$ is defined on $\left[\theta^{\omega},(0,1)\right] \subseteq \Theta$. Suppose $/ y / \geq \mu^{\prime}$. Then $y^{\prime} \in A_{\bar{n}}\left(R^{/ y /}\right)$ and so, by strategy-proofness, $\varphi_{\bar{n}}\left(R^{/ y /}\right)=y^{\prime}$. Otherwise, $/ y /<\mu^{\prime}$. That is, $R_{\bar{n}}^{\mu^{\prime}}=L^{\mu^{\prime}}$ with $\mu^{\prime}>/ y /=/ y^{\prime} /$, where $\alpha_{\bar{n}}^{l}\left(\mu^{\prime}\right)=y^{\prime}$. Thus, each $z \in A_{\bar{n}}\left(\mu^{\prime}\right)$ has $y^{\prime} \succ z$, and therefore $C\left(L^{\mu^{\prime}}, A_{\bar{n}}\left(\mu^{\prime}\right)\right)=\left\{y^{\prime}\right\}$, yielding $\varphi_{\bar{n}}\left(R^{\mu^{\prime}}\right)=y^{\prime}$. In sum, there is $\left.\left.\mu^{*} \in\left[\mu^{\prime}, \nu\right] \subseteq\right] \nu^{*}, \nu\right]$ such that $\varphi_{\bar{n}}\left(R^{\mu^{*}}\right)=y^{\prime}$. This implies by feasibility that $\varphi_{1}\left(R^{\mu^{*}}\right)<x$. However, $\mu^{*}>\nu^{*}$, so $x \in A_{1}\left(\mu^{*}\right)$, contradicting strategy-proofness. Conclude that $y \in A_{\bar{n}}(\mu)$.

Lemma 9. Assume that there is $p \in \mathbb{R}^{2}$ such that each $i \in N$ has $p \varphi_{i}(R)=$ $p \omega$ and, moreover, that no agent $i \in N$ has $\varphi_{i}(R)=\omega$. Assume also that for each $i \in N, \llbracket \varphi_{i}(R), \omega \rrbracket \subseteq A\left(R_{-i}\right)$. Choose $i \in N$ and let $R_{i}^{\prime}$ be such that $\varphi_{i}\left(R_{i}^{\prime}, R_{-i}\right) \in \llbracket \varphi_{i}(R), \omega \rrbracket$. Then it remains that each $j \in N$ has $p \varphi_{j}\left(R_{i}^{\prime}, R_{-i}\right)=p \omega, \llbracket \varphi_{j}\left(R_{i}^{\prime}, R_{-i}\right), \omega \rrbracket \subseteq A\left(R_{i}^{\prime}, R_{-i-j}\right)$, and no agent $j \in N$ has $\varphi_{j}\left(R_{i}^{\prime}, R_{-i}\right)=\omega$. 
Proof. Let $R_{i}^{\nu}$ be a path in $\mathcal{R}$ from $R_{i}$ to $R_{i}^{\prime}$ with $C\left(A\left(R_{i}\right), R_{i}^{\nu}\right) \subseteq \llbracket \varphi_{i}(R), \omega \rrbracket$. Let

$$
\nu^{*}=\inf \left\{\nu: \forall j \in N, \varphi_{j}\left(R_{i}^{\nu}, R_{-i}\right)=\omega\right\}
$$

and suppose there are $\nu^{\prime}<\nu^{*}$ and $j \in N$ with

$$
p \varphi_{j}\left(R_{i}^{\nu^{\prime}}, R_{-i}\right) \neq p \omega
$$

By feasibility there is $k \in N$ with $p \varphi_{k}\left(R_{i}^{\nu^{\prime}}, R_{-i}\right)>p \omega$. Since $p$ is normal to $\llbracket \varphi_{k}(R), \omega \rrbracket$, which is in $A\left(R_{-k}\right)$, to avoid a contradiction with Lemma 4. Lemma 7 requires that $\alpha_{k}^{r}\left(R_{i}^{\mu}, R_{-i-k}\right)$ or $\alpha_{k}^{l}\left(R_{i}^{\mu}, R_{-i-k}\right)$ first travel, along $\llbracket \varphi_{k}(R), \omega \rrbracket$, to $\omega$. By the convexity of preferences and the fact that $\varphi_{k}(R)$ is optimal for $R_{k}$ from $\llbracket \varphi_{k}(R), \omega \rrbracket$, there is $\nu^{\prime \prime}<\nu^{\prime}$ with $C\left(A\left(R_{i}^{\nu^{\prime \prime}}, R_{-i-k}\right), R_{k}\right)=\{\omega\}$. This violates strategy-proofness, since $\nu^{\prime \prime}<\nu^{*}$ implies $\varphi_{k}\left(R_{i}^{\nu^{\prime \prime}}, R_{-i}\right) \neq \omega$.

Conclude via continuity that for each $\nu^{\prime} \leq \nu^{*}$ and each $j \in N, p \varphi_{j}\left(R_{i}^{\nu^{\prime}}, R_{-i}\right)=$ $p \omega$. There is $j \in N$ such that $\varphi_{j}\left(R_{i}^{\nu^{*}}, R_{-i}\right)=\omega$. Since $i$ 's attainable set has not changed, $i$ is not consuming $\omega$ at this point, so there is at least one agent on each side of $\omega$. That is, there is $k$ with $\varphi_{k}\left(R_{i}^{\nu^{*}}, R_{-i}\right) \neq \omega$ and

$$
/ \varphi_{k}\left(R_{i}^{\nu^{*}}, R_{-i}\right) / \lessgtr / \omega / \lessgtr / \varphi_{i}\left(R_{i}^{\nu^{*}}, R_{-i}\right) /,
$$

where the top and bottom row of inequalities are two possible cases; the argument is similar for both. Prior to $\nu^{*}, j$ shares a side with either $k$ or $i$; assume it is $k$. Note that by Lemma 7 . $\llbracket \varphi_{k}(R), \omega \rrbracket \cap A_{k}\left(R_{i}^{\nu^{*}}, R_{-i-k}\right)$ has a non-trivial intersection. Thus there is a path, $R_{j}^{\mu}$, from $R_{j}$ to $R_{k}$ such that $C\left(\{y: p y=p \omega\}, R_{j}^{\mu}\right)$ remains on the same side of $\omega$ as $\varphi_{k 1}\left(R_{i}^{\nu^{*}}, R_{-i}\right)$. It follows that

$$
C\left(A\left(R_{i}^{\nu^{*}}, R_{-i-j}\right), R_{j}^{\mu}\right)=\{\omega\}
$$

and so of course this is the choice of the rule for $j$. Then by non-bossy nontraders, $\varphi_{k}\left(R_{i}^{\nu^{*}}, R_{j}^{1}, R_{-i-j}\right)=\varphi_{k}\left(R_{i}^{\nu^{*}}, R_{-i}\right) \neq \omega$, and we have a violation of anonymity. 
Thus, $\nu^{*}=\infty$, so for each element of the path and each $j \in N$, including $\nu=1, \varphi_{j}\left(R_{i}^{\nu}, R_{-i}\right) \neq \omega$. Since $[0,1]$ is compact, the distance from $\varphi_{j}\left(R_{i}^{\nu}, R_{-i}\right)$ to $\omega$ attains a minimum and thus is bounded away from zero on the entire path. By Proposition 3 and Lemma 7, there is a non-trivial $\llbracket z, \omega \rrbracket \subseteq \llbracket \varphi_{j}(R), \omega \rrbracket \subseteq A\left(R_{i}^{\prime}, R_{-i-j}\right)$. By the same arguments of the first paragraph, $p \varphi_{j}\left(R_{i}^{\nu}, R_{-i}\right)=p \omega$, and so we conclude that $\llbracket \varphi_{j}\left(R_{i}^{\nu}, R_{-i}\right), \omega \rrbracket \subseteq$ $A\left(R_{i}^{\prime}, R_{-i-j}\right)$.

Proof of Theorem 1. This now follows rather directly from Lemmas 8 and 9. The set of profiles $R$ such that

$$
\forall i, \forall k, C\left(\left\{x: q^{k} x=q^{k} \omega\right\}, R_{i}\right) \not \supset \omega
$$

is open and dense. We show the proof on this set and it follows on the whole domain by continuity.

Let $R$ be such a preference profile and let $N^{(1,0)}:=\left\{i \in N: p_{1}\left(R_{i} ; q^{k}\right)>\right.$ $\left.\omega_{1}\right\}$ have $\left|N^{(1,0)}\right|=k$. Relabel the agents so that $i \in N^{(1,0)}$ if and only if $L_{i}^{k}=L^{(1,0)}$. We show that for each $i \in N, q^{k} \varphi_{i}(R)=q^{k} \omega$. The proof is by induction. For each $\iota \in N$, let $R^{\iota}:=\left(R_{1}, \ldots, R_{\iota}, L_{\iota+1}^{k}, \ldots, L_{|N|}^{k}\right)$. Induction will be with respect to $\iota$.

If $\varphi_{1}\left(L^{k}\right)=\omega$, then all agents consume $\omega$, and the result follows directly from non-bossy non-traders.

By Lemma 8, for each $i \in N, \llbracket \varphi_{i}\left(R^{0}\right), \omega \rrbracket \subseteq A\left(R_{-i}^{0}\right)$. Since $R$ is a generic economy, $C\left(\llbracket \varphi_{1}\left(R^{0}\right), \omega \rrbracket, R_{1}\right) \not \ngtr \omega$, so Lemma 9 can be invoked. Repeating this yields the result.

\section{REFERENCES}

BARBERÀ, S., AND M. O. JACKSON (1995): "Strategy-proof exchange," Econometrica, 63, 51-87, 10.2307/2951697.

Hildenbrand, W. (1970): "On economies with many agents," Journal of Economic Theory, 2, 161-188.

(1974): Core and Equilibria of a Large Economy.: Princeton University Press. 
Kannai, Y. (1970): "Continuity properties of the core of a market," Econometrica, 38, 791-815.

Kelley, J. L. (2008): General Topology: Ishi Press International, New York.

Mount, K. R., And S. Reiter (1996): "A lower bound on computational complexity given by revelation mechanisms," Economic Theory, 7, 237266, 10.1007/BF01213904.

Nisan, N., AND I. SEgal (2006): "The communication requirements of efficient allocations and supporting prices," Journal of Economic Theory, 129, 192-224, 10.1016/j.jet.2004.10.007.

SERIzaWA, S., AND J. A. Weymark (2003): "Efficient strategy-proof exchange and minimum consumption guarantees," Journal of Economic Theory, 109, 246-263, 10.1016/S0022-0531(03)00019-X.

Sprumont, Y. (1991): "The division problem with single-peaked preferences: A characterization of the uniform allocation rule," Econometrica, 59, 509-519, $10.2307 / 2938268$.

(1995): "A note on strategyproofness in Edgeworth-Box economies," Economics Letters, 49, 45-50, 10.1016/0165-1765(94)00646-J.

You, J. S., AND R. JuAREz (2021): "Incentive-compatible simple mechanisms," Economic Theory, 10.1007/s00199-021-01342-z.

Zhou, L. (1991): "Inefficiency of strategy-proof allocation mechanisms in pure exchange economies," Social Choice and Welfare, 8, 10.1007/ BF00177662,

Department of Business and Economics, University of Southern Denmark, Campusvej 55, Odense M, Denmark 5230 